\title{
DISABILITY AND MEDICAL REHABILITATION. ANALYSIS OF BARRIERS AND RECOMMENDATIONS PROPOSED BY THE WORLD HEALTH ORGANIZATION
}

\author{
Dogaru Gabriela $^{12}$; Stanescu Ioana1 ${ }^{1}$; Pasca Luminita ${ }^{13}$; Aluas Maria1 \\ 1."Iuliu Hatieganu" University of Medicine and Pharmacy Cluj-Napoca \\ 2.Clinical Rehabilitation Hospital Cluj-Napoca \\ 3.City Clinical Hospital Cluj-Napoca
}

\section{Defining the problem.}

Disability is part of human condition. Anyone can suffer at a certain point in life a temporary or permanent disability. Disability refers to difficulties found in any of the three areas of functioning and is the term for impairments, activity limitations, and participation restrictions, which refer to the negative aspects of the interaction between an individual (with a certain health condition) and the contextual factors of that individual (personal factors motivation, self-esteem, and environmental factors - products and technology, service systems, policies, relations with the others). The concept of disability is related to human rights because: people with disabilities are confronted with injustice when they are denied employment, education, political participation; they are subject to violence, abuse, prejudice, disrespect. Medical rehabilitation measures are aimed at functions and structures of the body, activities and social participation, environmental and personal factors. The results of rehabilitation are the benefits and changes in the functioning of an individual during the course of time, which can be attributed to a single measure or to a set of measures (1).

\section{Presentation of the problem}

The World Report on Disability was launched by the World Health Organization and the World Bank and it brings a major contribution to the understanding of disability and its impact on individuals and society. It was issued for four years, with the participation of 360 researchers, some of which were confronted with various disabilities, with the consultation of 74 countries. It is considered an approach intended to offer innovative solutions, policies and programs to improve the daily life of people with disabilities. It helps implement the rights established by the United Nations Convention on the Rights of Persons with Disabilities, which came into force in May 2006 and was ratified by Romania in 2010 . It is intended for medical rehabilitation doctors, university teaching staff, researchers, political decision making factors, civil society. The general objectives are to provide governments and the civil society with an elaborate description of the importance of disability, based on the best scientific information available; to make recommendations regarding the actions to be undertaken at national and international level $(1,2)$.

More than one billion people worldwide live with a form of disability, which represents about $15 \%$ of the world population, according to global population estimates of 2010. Over 190 million people are confronted with severe disabilities such as tetraplegia, severe depression or blinding, according to the Global Burden of Disease Study, ordered by the World Bank (1). The estimated number of children (0-14 years) who live with a disability varies between 93 millions and 150 millions. According to the Directorate for the Protection of Persons with Disabilities (DPPH), in 2012 there were 690,000 people with disabilities in Romania, including children and adults, which means that $4 \%$ of the population had 
a form of disability. Of the 611,000 adult non-institutionalized persons with disabilities, in 2012 only 27,000 persons were employed in Romania (3).

The World Report on Disability attempts to offer solutions to facilitate the implementation of the United Nations Convention on the Rights of Persons with Disabilities (UNCRPD). It documents the living conditions of people with disabilities throughout the world and explores the measures that can facilitate their participation to society, health, rehabilitation, education, employment. It addresses disability from the perspective of human rights, the intersection between disability and personal development (1).

The International Classification of Functioning, Disability and Health (ICF, 2001, 2006) was adopted as a general conceptual framework for this report. Within ICF, the problems related to human functioning are divided into 3 interconnecting areas $(1,4)$ :

-impairments are problems of the body functioning or changes of the body structure, e.g. paralysis or blinding

-activity limitations represent difficulties in performing activities, e.g. walking, eating

\section{-participation restrictions -}

confrontation with discrimination at the workplace or in public transportation means

More than 40 countries adopted the legislation against the discrimination of persons with disabilities in the 1960s. The CRPD adopted in 2006 outlines the civil, social, economic rights of persons with disabilities. The basic message is that people with disabilities should not be considered as "objects" to manage, but as "subjects" that deserve equal respect, as well as the respect of human rights (1).

In Australia, a survey of the population with disabilities carried out in 1998 showed that the most common disorders associated with disabilities were arthrosis, dorsolumbalgia, hearing deficiencies, arterial hypertension (AHT), heart disease, hearing loss, diabetes, cerebrovascular accidents (CVA), depression, dementia (1). An American study performed in 2001, regarding the 10 most frequent conditions associated with disability, showed that rheumatism was the main cause among elderly people, accounting for $30 \%$ of adults aged over 65 years who reported limitations of their daily activities. 23\% had cardiac disorders, which ranked second. The other disabling conditions were AHT, spinal disorders, diabetes, fractures, CVA, impairment of sight, hearing, respiratory problems (1).

\section{The general health care system}

Article 25 of the United Nations Convention on the Rights of Persons with Disabilities reinforces the right of these persons to benefit from the highest health care standards, without discrimination. An increasing number of studies suggest that people with disabilities as a group have a poor health status compared to the rest of the population. Depression is a secondary condition common in disabled people. Pain has been reported in children and adults who suffer from cerebral palsy, in those with spina bifida, and in adults with postpoliomyelitis paralysis, neuromuscular disease, and cerebral lesions. Osteoporosis is more frequent among people with spinal cord lesions, spina bifida, cerebral palsy. The aging process starts earlier than usual, some persons with disabilities present premature aging signs at 40 or 50 years and are most frequently confronted with health conditions associated with age. An Australian study shows that disabled people aged between 15-64 years are more likely to be overweight or obese than the rest of the population (48\% compared to $39 \%)$ and to smoke daily (1).

Persons with disabilities encounter a series of barriers when they attempt to gain access to health care services. Reforms are needed in the following areas:

1. Approach of policies and legislation

2. Approach of barriers to financing and financial availability 


\section{Approach of barriers to health care services}

4. Approach of barriers to human resources

5. Filling of data and research gaps

Health policies, international and national legislation can help meet the health care needs of people with disabilities, when there is a political will. CRPD indicates the following action areas:

Accessibility to the health care system, health care services, health insurance, environmental accessibility. Financial accessibility - making sure that disabled persons benefit from the same variety, quality and standard, and financial accessibility of health care services as the rest of people. Availability - making available early intervention and treatment services as close as possible to the place where people live in their communities. Quality - making sure that the personnel involved provides the same quality of health care services for disabled persons as for persons without disabilities (1).

It is recommended to change legislation and policies in the field of education, transportation, accommodation, employment, social ensurance, which can influence the health of persons with disabilities.

A smaller number of employed people with disabilities is reported, because they are economically disadvantaged and consequently, less likely to afford public, private or combined health insurance, which would increase the access of people to health care services.

It is important to ensure the availability of health care services and to increase the awareness of disabled people about the existence of these services, including in rural or isolated areas. Where health care services are available, persons with disabilities may encounter a series of physical, communication, information and coordination barriers. Physical barriers are most frequently related to the architectural design of the institution or to medical equipment or transportation. Most barriers include inaccessibility to parking spaces, buildings, insufficient signing, narrow entrances, interior staircases without elevators, no toilet facilities. Many women in wheelchairs cannot have access to tests for the detection of breast and cervical cancer, because the height of examination tables cannot be adjusted, and the mammography equipment can only be used in a standing position. Disabled persons often report that transportation is a barrier to access to the health care system, particularly when they are at a distance from medical facilities (1).

In India, a national program was implemented between 1999 and 2004 by the Rehabilitation Council, in order to train doctors in primary health care centers to provide care for persons with locomotor disabilities. The objectives included the dissemination of knowledge on prevention, health promotion, treatment and medical rehabilitation, the increase of the awareness of medical authorities about general topics related to disability such as legislation and human rights (1).

Article 26 on Habilitation and Rehabilitation of the United Nations Convention on the Rights of Persons with Disabilities (CRPD) requires "...appropriate measures, including through peer support, to enable persons with disabilities to attain and maintain maximum independence, full physical, mental, social and vocational ability, and full inclusion and participation in all aspects of life" (2). This Report defines rehabilitation as "a set of measures to assist individuals that are or may be confronted with disabilities in order to reach and maintain an optimal functioning in the interaction with their environments". Rehabilitation is aimed at improving individual functioning, for example, by improving the capacity of a person to eat and drink independently. Rehabilitation also includes changes in the individual environment, for example, by installing a handrail in toilets (1). 
According to this report, the barriers to medical rehabilitation services can be overcome through a series of actions, such as: reform of policies, laws and offering systems, including the development and revision of national rehabilitation plans; development of financing mechanisms to overcome barriers related to the financing of rehabilitation; increase of human resources for rehabilitation, including the training and maintenance of rehabilitation personnel; extension and decentralization of service provision; increase of the use of and accessibility to assistive technology and devices; extension of research programs, including the improvement of information and access to good practice principles (1).

Development of community rehabilitation and home care

Community based rehabilitation. In many countries with low and medium income, community based rehabilitation (CBR) programs provided by the state or NGOs become a source of assistance and support for many disabled people and their families. Many are focused on the provision of information, work with the families, and the facilitation of the participation of persons with disabilities in the community. RUCODE, an NGO from the Tamil Nadu State of India, offers community day centers for children with intellectual impairment and cerebral palsy, with the help of local administration and parents. Each center takes care of approximately 10 children, with a teacher and an assistant in each center. The community contributes the location and provides lunch for the children (1). Community based home care. Community based home care is any kind of support provided for sick persons and their families at their homes. In many countries, there are governmental community based home care programs, involving the provision of food, transportation, drugs, temporary care, money allocations.

Recommendations proposed by the World Report on Disability
Recommendation 1: Allowing access to all conventional policies, systems and services. Integration is the process by which governments and other decision making factors make sure that persons with disabilities participate on a basis of equality with other persons in any activity and service intended for the general public, such as education, health, employment and social services. Barriers to participation should be removed, possibly with changes of the laws, policies, institutions and environments.

Recommendation 2: Investing in programs and services specific for persons with disabilities. In addition to integrated services, some people may need access to specific measures, such as rehabilitation, support services or training. Rehabilitation - including assistive technologies such as wheelchairs, hearing aids and white canes, improves functioning and independence.

Recommendation 3: Adopting a national strategy and an action plan. It is also recommended to adopt a national strategy and a national action plan for disability. A national disability strategy establishes reinforced and detailed longterm measures for the improvement of the welfare of persons with disabilities and must cover both policies and services specific for the disabled.

Recommendation 4: Implication of persons with disabilities. Persons with disabilities frequently have a unique way to view their own disability and situation. Disabled people should be consulted and actively involved in the formulation and implementation of policies, laws and services.

Recommendation 5: Improving the skills of human resources. Disability training, which integrates the principles of human rights, should be included in current programs and in accreditation programs.

Recommendation 6: Providing adequate financing and improving financial accessibility. The promotion of public-private partnerships, particularly for 
non-profit organizations, and decentralization in favor of persons with disabilities for a consumer oriented health care approach may contribute to the improvement of services.

Recommendation 7: Increasing public awareness and understanding

Recommendation 8: Improving the collection of data on disability. Uniform definitions referring to disability, based on ICF, can allow the presence of comparable data at international level.

Recommendation 9: Development and support of research on disability. Research is essential for a better public understanding of problems related to disability, for the information about disability policies and problems and the allocation of sufficient resources (1).

\section{Conclusions}

The World Report on Disability presented the current situation of persons with disabilities. It evidenced the knowledge gaps and emphasized the need for further studies and policy elaboration. It also provided recommendations for the construction of an inclusive and permissive society, supporting the equality of chances in order for each person with disabilities to reach their potential. The adoption of a national strategy and a national action plan for patients with disabilities is required. A national strategy with reinforced and detailed long-term measures for the increase of the welfare of persons with disabilities should cover both the policies and services specific for the disabled. Persons with disabilities should be consulted and actively involved in the formulation and implementation of policies, laws and services.
References

1. Raport mondial privind dizabilitatea. Bucuresti, 2012

2. Convention on the Rights of Persons with Disabilities. New York, United Nations, 2006

3. www.anph.ro/legislatie, accessed in May 2012

International Classification of Functioning, Disability and Health (ICF). Geneva, World Health Organization, 2001 